\title{
The clinical implications of G1-G6 transcriptomic signature and 5-gene score in Korean patients with hepatocellular carcinoma
}

Sung-Min Ahn ${ }^{1,2+}$, Farhan Haq ${ }^{3 \dagger}$, Inkeun Park ${ }^{1}$, Jean-Charles Nault ${ }^{4,5,6,7}$, Jessica Zucman-Rossi ${ }^{4,5,6,7^{*}}$ and Eunsil $\mathrm{Yu}^{8^{*}}$

\begin{abstract}
Background: Efforts have been made to classify Hepatocellular Carcinoma (HCC) at surgically curable stages because molecular classification, which is prognostically informative, can accurately identify patients in need of additional early therapeutic interventions. Recently, HCC classification based French studies on the expression of 16 genes and 5 genes were proposed. In 16-gene classification, transcriptomic signatures (G1-G6) were used to classify HCC patients into clinical, genomic and pathway-specific subgroups. In 5-gene score classification, the good or poor prognosis of HCC patients was predicted. The patient's cohort in these studies was mainly from Caucasian and African populations. Here, we aimed to validate G1-G6 and 5-gene score signatures in 205 Korean HCC patients since genomic profiles of Korean patients are distinct from other regions.

Methods: Integrated analyses using whole-exome sequencing, copy number variation and clinical data was performed against these two signatures to find statistical correlations. Kaplan-Meier, univariate and multivariate COX regression analysis were performed for Disease-Specific Survival (DSS) and Recurrence-Free Survival (RFS).

Results: The G2 and G3 subgroups of transcriptomic signature were significantly associated with TP53 mutations while G5 and G6 subgroups were significantly associated with CTNNB1 mutations which is in concordance with original French studies. Similarly, the poor prognosis group of 5-gene score showed shorter DSS $(p=0.045)$ and early RFS $(p=0.023)$ as well as a significant association with microvascular invasion, tumor size $(>5 \mathrm{~cm})$, elevated AFP levels, and RB1 mutations. However, the 5-gene score was not an independent prognostic factor for survival.

Conclusion: The G1-G6 and 5-gene signatures showed significant concordance between genetic profiles of Korean HCC patients and patients in original French studies. Thus, G1-G6 and 5-gene score signatures can be targeted as potential therapeutic biomarkers against HCC patients worldwide.
\end{abstract}

Keywords: 5-gene score, G1-G6 subgroups, Survival, Prognosis

\footnotetext{
* Correspondence: jessica.zucman-rossi@inserm.fr; esyu@amc.seoul.kr

${ }^{\dagger}$ Sung-Min Ahn and Farhan Haq contributed equally to this work.

${ }^{4}$ Inserm, UMR-1162, Génomique Fonctionnelle des Tumeurs Solides, Équipe

Labellisée Ligue Contre le Cancer, 27 rue Juliette Dodu, F-75010 Paris, France

${ }^{8}$ Department of Pathology, University of Ulsan College of Medicine, Asan

Medical Center, 88, OLYMPIC-RO 43-GIL, SONGPA-GU, SEOUL 138-736, South

Korea

Full list of author information is available at the end of the article
}

(c) The Author(s). 2018 Open Access This article is distributed under the terms of the Creative Commons Attribution 4.0 International License (http://creativecommons.org/licenses/by/4.0/), which permits unrestricted use, distribution, and reproduction in any medium, provided you give appropriate credit to the original author(s) and the source, provide a link to the Creative Commons license, and indicate if changes were made. The Creative Commons Public Domain Dedication waiver (http://creativecommons.org/publicdomain/zero/1.0/) applies to the data made available in this article, unless otherwise stated. 


\section{Background}

Hepatocellular carcinoma is the most common type of liver cancer worldwide [1]. Liver resection is one of the most viable treatment option for HCC patients, but associated with high risk of recurrence [2, 3]. In advanced HCC, no clinical trial studies have convincingly improved survival, except for the sorafenib trial [4]. The failure of these trials is partly due to the lack of effective molecular markers or the minimal validation of known molecular markers in diverse multi-ethnic populations. Efforts have been made to classify HCC at surgically curable stages because molecular classification, which is prognostically informative, can accurately identify patients in need of additional early therapeutic interventions [5-9].

In HCC, several molecular classification-based microarray studies have been reported. In the first two studies, HBV-positive HCC patients from Belgium and China were classified into good or poor prognosis groups $[10,11]$. The expression of hepatoblast-related genes was significantly associated with poor prognosis of HCC patents [11]. In another HCC study using a Caucasian population of HCC patients, a gene signature of 186 genes was identified in non-tumor liver, out of which 113 showed good prognosis and 73 showed poor prognosis [12].

Recently, HCC classification methods based on the expression of 16 genes and 5 genes were proposed $[13,14]$. In the 16-gene based classification, transcriptomic signatures (G1-G6) were used to classify HCC patients into clinical, genomic and pathway-specific G1-G6 subgroups [13]. In the 5-gene score-based classification, the good or poor prognosis of HCC patients was predicted [14]. The study showed the efficacy of 5-gene score as a potential biomarker in American, Caucasian and Chinese HCC patients [14].

In this study, we aimed to validate the clinical relevance of the 16-gene and 5-gene score methods in a Korean population. One of the key motivations of this study was that the genomic profiles of HCC patients were distinct between Western and Korean populations. For example, the TP53 mutation rate is very high in Korean populations, but unlike African or Chinese populations, the TP53 R249S mutation rate is almost zero and has no association with tumor recurrence and survival $[5,8,15,16]$. In addition, genetic aberrations in $R B 1$ are associated with poor prognosis in Korean HCC patients, which has not been observed in Western populations $[5,8]$. In this study, we generated the transcriptomic signatures using the 16-gene and 5-gene score methods, and analyzed them with the genomic profiles obtained by whole-exome sequencing and clinicopathological features of the HCC cases used.

\section{Methods}

Study design and clinical samples

Two hundred five Fresh frozen tissues of HCC patients at a surgically curable stage were used in this study. The surgically curable stage was defined by Milan criteria which clearly excludes extra hepatic metastasis and macrovascular invasion. Patients with either one of them cannot have surgical resection. The institutional review board of ASAN, South Korea, approved all of the samples, along with documented consent from all patients who participated in the study (2012-0389). The estimated tumor cellularity of each sample was more than $70 \%$. Clinical features include tumor size, microvascular invasion, recurrence, the Edmondson-Steiner histological grade, the fibrosis stage of the nonneoplastic liver tissue, viral infection, tumor nodules (monofocal vs multifocal) and the serum alphafetoprotein levels (Table 1 ).

Table 1 Patients and tumor characteristics

\begin{tabular}{ll}
\hline Characteristics & Frequencies \\
\hline Age at Surgery (Median, Range) & $55(26-80)$ \\
$<60$ years & $137(66.83 \%)$ \\
$>60$ years & $68(33.17 \%)$ \\
Etiology of the liver disease & \\
HBV & \\
HCV & $148(72.20 \%)$ \\
NBNC & $20(9.76 \%)$ \\
Tumor size (median, range) & $37(18.05 \%)$ \\
$<5$ cm & $3.8(1.2-16)$ \\
$>5$ cm & $140(68.29 \%)$ \\
Microvascular invasion & $68(33.17 \%)$ \\
Yes & \\
No & $59(28.78 \%)$ \\
Serum AFP level (median, range) & $146(71.22 \%)$ \\
$<20$ & $42.7(0.76-472,000)$ \\
$>20$ & $91(44.39 \%)$ \\
Hepatic fibrosis stage & $114(55.61 \%)$ \\
$1,2,3$ & $137(66.83 \%)$ \\
4 & $68(33.17 \%)$ \\
Edmondson-Steiner grade & \\
Tumor nodules & $193(94.15 \%)$ \\
Multiple & $96(46.83 \%)$ \\
\hline
\end{tabular}

${ }^{a} \mathrm{HBV}$ : hepatitis $B$ virus.

bHCV: hepatitis $C$ virus.

${ }^{c} N B N C$ : non-hepatitis B, non-hepatitis C 
Whole exome sequencing and copy number variation (CNV) analysis

As mentioned in our previous study, DNeasy Blood and Tissue kit (Qiagen) were used for DNA extraction from the tumor tissues. Exome sequencing was done using Illumina HiSeq 2000 platform [5]. The sequenced reads were aligned to the UCSC hg19 release of the human genome. Somatic mutations in TP53, CTNNB1 and RB1 were identified using MuTect [17]. CNV analysis was performed using the Affymetrix Cytoscan HD platform. CNV data were analysed with the Nexus Copy Number software (BioDiscovery, CA, ver. 6.1). Furthermore, $\mathrm{LOH}$ events were identified using PSCBS algorithm [18].

\section{G1-G6 classification and 5 gene score prediction}

The detailed information about the development of G1G6 and 5-gene score method was described in previous HCC studies [13, 14]. Briefly, the G1-G6 classification was done using robust unsupervised hierarchical clustering using 6712 probes of Affymetrix HG-U133A GeneChip ${ }^{\text {Tx }}$ [13]. The stability and reproducibility of the clusters were carefully evaluated. The mean reproducibility of all groups was more than $90 \%$. On the basis of clusters, tumors were classified into G1-G6 subgroups. Furthermore, using quantitative RT-PCR data and applying 5 prediction algorithms (including SVM, PAM, kNN, DQDA, DLDA) on 103 genes associated with the prognosis and diagnosis of cancer, 16 genes were identified which properly classified HCC patients into G1-G6 subgroups. Similarly, univariate Cox model was generated for each of the 103 genes against prognosis and survival of HCC patients. 31 significant genes were further optimized to only 5 genes using multivariate COX model [14]. In this study, we evaluated the performance of G1-G6 and 5-gene scores in Korean population using high quality RNA data 205 of HCC patients.

\section{Statistical analysis}

We performed several statistical analyses against these two molecular markers to establish their clinical and pathological relevance in a Korean population. IBM SPSS Version 20 was used for all statistical analyses. Fisher's exact test $(P<0.05)$ was used to calculate any association between the genomic data and the G1-G6 and 5-gene score. Kaplan-Meier survival analysis was performed using DSF and RFS data. Previously, early recurrence was defined as recurrence before 24 months [14]. However, in our study recurrence before 12 months was considered as early recurrence. Univariate and multivariate Cox regression survival analyses were performed to validate the prognostic association between clinical data, genomic data and G1-G6 and 5-gene score.

\section{Results and discussion}

Out of 231 cases of HCC used in previous study, we were able to extract high-quality RNA of 205 HCC cases [5]. We then found the association of G1-G6 subgroups and 5-gene score with mutations, CNVs and clinicopathological features (see Table 1 for clinical features).

\section{Validation of the G1-G6 classification in Korean population}

Out of the 205 cases analyzed, 16 (7.81\%) fell into G1, 23 (11.22\%) into G2, 16 (7.81\%) into G3, 110 (53.65\%) into G4, 27 (13.17\%) into G5 and 13 (6.34\%) into G6. The occurrence of the G1-G3 and G5-G6 subgroups in a previous French study were slightly higher compared to our dataset, except for the G4 subgroup, which accounted for the largest proportion in both studies (Fig. 1) [13, 14]. Of note, there is no significant difference between G1-G6 distribution in both cohorts (Mann-Whitney test, $P=0.297$ ). In addition, the two cohorts were strongly correlated (Spearman Correlation $=0.89, P=0.033$ ).

\section{Molecular and clinicopathologic characteristics correlated with the G1-G6 classification}

Then, we analyzed the G1-G6 subgroups with genetic aberrations and the clinical features of the 205 cases of HCC. G2 and G3 were significantly associated with TP53 mutations $(P<0.005)$ and G5 and G6 with CTNNB1 mutations $(\mathrm{P}<0.005)$. Of note, almost all of the G6 subgroup cases $(12 / 13)$ harbored somatic CTNNB1 mutations. The associations between G2-G3 and TP53 mutations and between G5-G6 and CTNNB1 mutations in this study were consistent with the original observations made in the French study [13].

As for CNVs, G2 was significantly associated with $13 \mathrm{q}$ LOH $(P<0.05)$; G2-G3 with $17 \mathrm{p} \mathrm{LOH}(P<0.05)$; and G1-G3 with 4q $(P<0.001), 5 \mathrm{q}(P<0.001)$ and $16 \mathrm{p} \mathrm{LOH}$ $(P<0.001)$. All of these LOH events were associated with G1-G3, which are collectively recognized as HCC subgroups with chromosomal instability [13]. Again, the

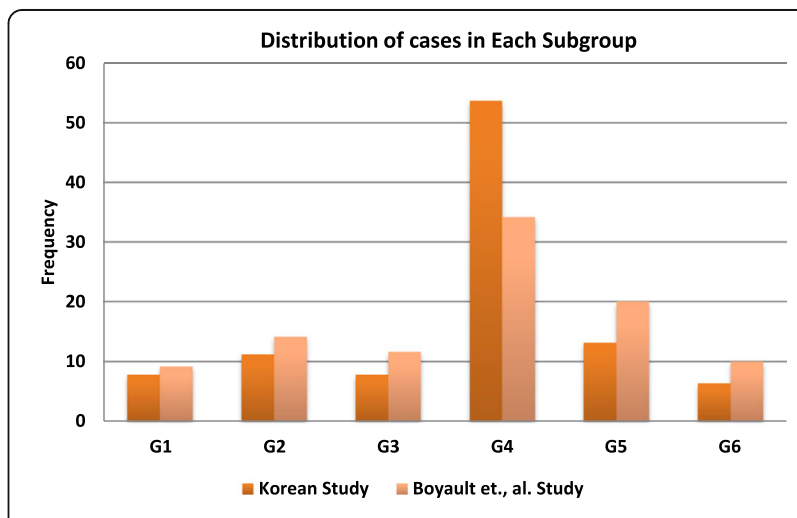

Fig. 1 Distribution of G1-G6 subgroups in French and Korean studies 
observations in this study were consistent with the original observations made in the French study [13].

As for clinicopathological features, G1-G3 groups were significantly associated with AFP $>100 \mathrm{IU} / \mathrm{ml}(P<0.001)$, whereas, G4-G6 were significantly associated with AFP $<100 \mathrm{IU} / \mathrm{ml}(P<0.001)$. G5 was significantly associated with tumor size $(>5 \mathrm{~cm})(P<0.05)$, which was not observed in the previous study. When we performed a survival analysis, the G1-G6 subgroups did not show any significant difference in either DSS or RFS (i.e., recurrence before 12 months), which was consistent with the French study [13].

\section{5-gene score and its role in prognostication}

To validate the 5 -gene score, we classified patients into a good prognosis group (81 cases, $40 \%$ ) and a poor prognosis group (124 cases, $60 \%$ ) according to the 5-gene score and evaluated the difference in DSS and RFS. As demonstrated in Fig. 2, the poor prognosis group showed a shorter median DSS $(P<0.05)$ and RFS $(P<0.05)$.

\section{Molecular and clinicopathologic features correlated with the 5-gene score}

We found that the poor prognosis group, as predicted by the 5 -gene score, was significantly associated with TP53 mutations $(P<0.005)$. This association needs to be interpreted with caution. In our previous study, we reported that TP53 mutations were not associated with poor survival in a Korean population; however, in other studies, TP53 mutations, especially the R249S mutation resulting from aflatoxin B1 exposure, were associated with poor survival in HCC patients. In the 205 cases of HCC that we used in this study, no case harbored the TP53 R249S mutation. However, in the French study, 12 out 62 patients showed R249S mutations, all in migrants from Africa or Asia, but did not show any association with either survival or poor prognosis as predicted by the 5-gene score [14].

In addition, in our recent HCC study, we reported that RB1 aberrations (Homozygous Deletions and Inactivating Mutations) were associated with the poor prognosis of HCC patients after resection. Consistent with previous observations, the poor prognosis predicted by the 5gene score also showed association with $R B 1$ aberrations (i.e., 13 out of 18 (72\%) cases fell into the poor prognosis group). In addition, the poor prognosis group was also significantly associated with a loss of heterozygosity $(\mathrm{LOH})$ events at the $4 \mathrm{q}, 5 \mathrm{q}, 16 \mathrm{p}, 17 \mathrm{p}$ and $22 \mathrm{q}$ chromosomal arms, which was also consistent with the French study $(P<0.05)[13]$.

As for clinical features, the poor prognosis group was significantly associated with microvascular invasion $(P<0.005)$, tumor size $(>5 \mathrm{~cm})(P<0.05)$, and high AFP levels $(>20 \mathrm{ng} / \mathrm{ml}) \quad P<0.005)$. In addition, we found that the poor prognosis group was significantly associated with G1-G3 and the good prognosis group with G4-G6 $(P<0.005)$.

\section{Univariate and multivariate analysis for disease-specific survival (DSS)}

With a median follow-up period of 53.3 months (range 5.5-88.9 months), 30 patients died due to HCC. The two- and five-year DSS rates were $94.5 \%$ and $79.9 \%$, respectively. In the univariate analysis, DSS was significantly associated with the 5-gene score (hazard ratio (HR) 2.381, 95\% confidence interval (CI) 1.021-5.56, $P=0.045$ ), tumor nodules (HR 8.69, 95\% CI 3.84619.6653, $P<0.001$ ), microvascular invasion (HR 2.496, 95\% CI 1.218-5.118, $P=0.012$ ), tumor size (HR 2.674, 95\% CI 1.297-5.512, $P=0.008)$ and $R B 1$ aberrations

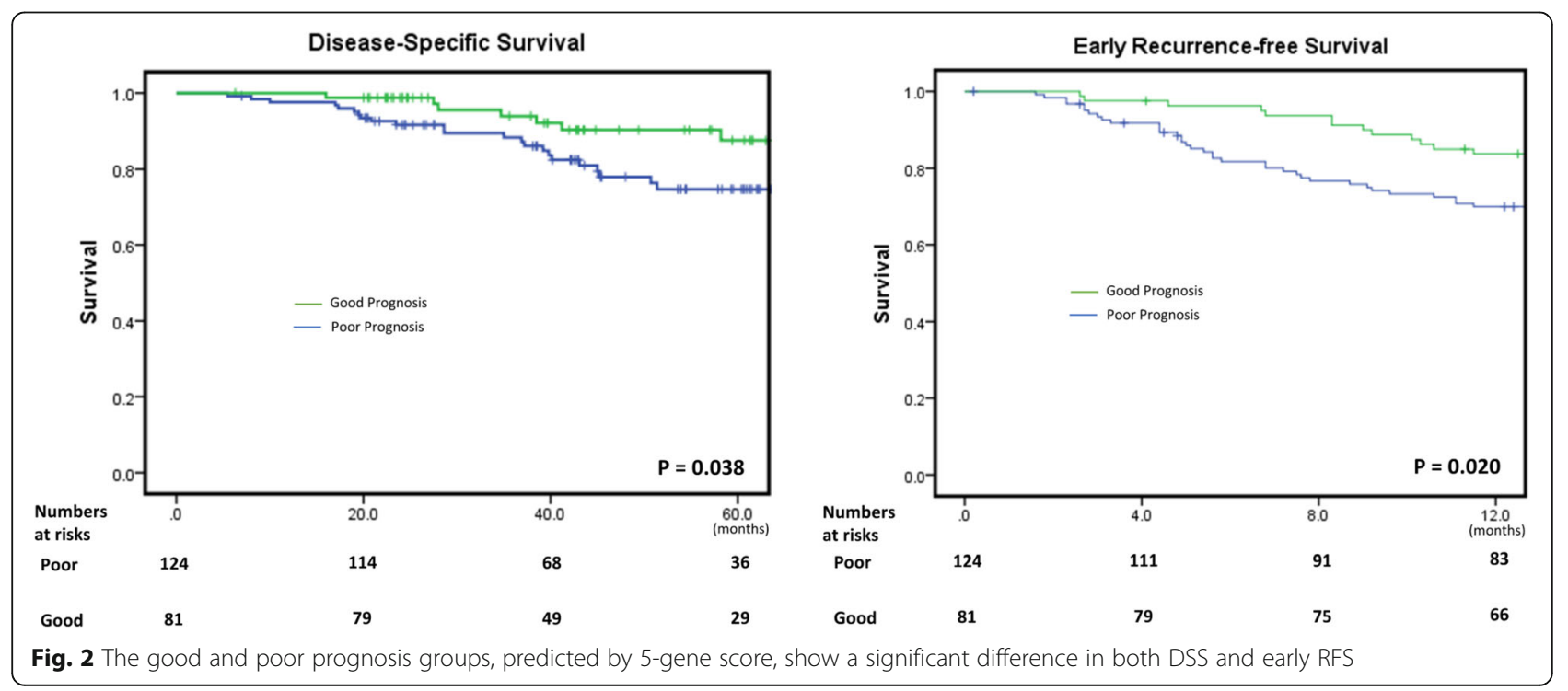


Table 2 Cox regression analysis for DSS in 205 patients with resectable HCC

\begin{tabular}{|c|c|c|c|c|c|c|c|c|}
\hline & \multicolumn{4}{|c|}{ Univariate analysis } & \multicolumn{4}{|c|}{ Multivariable analysis } \\
\hline & \multirow[b]{2}{*}{$P$-value } & \multirow[b]{2}{*}{$\mathrm{HR}$} & \multicolumn{2}{|c|}{$95.0 \% \mathrm{Cl}$} & \multirow[b]{2}{*}{$P$-value } & \multirow[b]{2}{*}{$\mathrm{HR}$} & \multicolumn{2}{|c|}{$95.0 \% \mathrm{Cl}$} \\
\hline & & & Lower & Upper & & & Lower & Upper \\
\hline 5-gene score (poor vs. good group) & 0.045 & 2.381 & 1.021 & 5.551 & 0.278 & 1.368 & 0.518 & 3.614 \\
\hline Age $(>=60$ vs. $<60)$ & 0.867 & 1.033 & 0.706 & 1.511 & & & & \\
\hline HBV & 0.593 & 0.813 & 0.380 & 1.737 & & & & \\
\hline $\mathrm{HCV}$ & 0.076 & 1.548 & 0.956 & 2.507 & & & & \\
\hline NBNC & 0.547 & 0.906 & 0.658 & 1.249 & & & & \\
\hline Tumor nodules & 0.000 & 8.697 & 3.846 & 19.665 & 0.000 & 8.411 & 3.263 & 21.68 \\
\hline Cirrhosis (Yes vs. No) & 0.581 & 1.225 & 0.597 & 2.515 & & & & \\
\hline VI (Yes vs. No) & 0.012 & 2.496 & 1.218 & 5.118 & 0.580 & 1.260 & 0.556 & 2.856 \\
\hline Tumor size ( $>=5 \mathrm{~cm}$ vs $<5 \mathrm{~cm}$ ) & 0.008 & 2.674 & 1.297 & 5.512 & 0.026 & 2.534 & 1.116 & 5.755 \\
\hline Edmonson Grade & 0.226 & 1.254 & 0.87 & 1.808 & & & & \\
\hline AFP level (Normal $(<20)$ vs. elevated) & 0.092 & 1.956 & 0.896 & 4.272 & & & & \\
\hline RB1 mutation & 0.008 & 3.133 & 1.344 & 7.303 & 0.527 & 1.368 & 0.518 & 3.614 \\
\hline TP53 mutation & 0.629 & 0.819 & 0.364 & 1.841 & & & & \\
\hline CTNNB1 mutation & 0.212 & 0.542 & 0.207 & 1.418 & & & & \\
\hline
\end{tabular}

*VI Microvascular Invasion

(HR 3.133, 95\% CI 1.344-7.303, $P=0.008$ ) (Table 2). In the multivariable analysis, the 5-gene score was not validated as a significant prognostic factor; tumor nodules (HR 8.411, CI 3.263-21.681, $P<0.001$ ) and tumor size (HR 2.534, CI $1.116-5.755, P=0.026$ ) were the significant prognostic factors for the survival of $\mathrm{HCC}$ patients after resection (Table 2 ).
Univariate and multivariable analysis for early recurrencefree survival (RFS)

During follow-up, 49 out of 205 patients had disease recurrence within 12 months. In the univariate analysis, the early RFS was significantly associated with the 5-gene score (HR 2.087, 95\% CI 1.106-3.936, $P=0.023$ ), tumor nodules (HR 5.308, 95\% CI 2.474-11.390, $P<0.001$ ),

Table 3 Cox regression analysis for eary RFS in 205 patients with resectable HCC

\begin{tabular}{|c|c|c|c|c|c|c|c|c|}
\hline & \multicolumn{4}{|c|}{ Univariate analysis } & \multicolumn{4}{|c|}{ Multivariable analysis } \\
\hline & \multirow[b]{2}{*}{$P$-value } & \multirow[b]{2}{*}{$\mathrm{HR}$} & \multicolumn{2}{|c|}{$95.0 \% \mathrm{Cl}$} & \multirow[b]{2}{*}{$P$-value } & \multirow[b]{2}{*}{$H R$} & \multicolumn{2}{|c|}{$95.0 \% \mathrm{Cl}$} \\
\hline & & & Lower & Upper & & & Lower & Upper \\
\hline 5-gene score (poor vs. good group) & 0.023 & 2.087 & 1.106 & 3.936 & 0.361 & 1.373 & 0.696 & 2.708 \\
\hline Age $(>=60$ vs. $<60)$ & 0.469 & 1.113 & 0.833 & 1.488 & & & & \\
\hline HBV & 0.814 & 1.079 & 0.572 & 2.035 & & & & \\
\hline $\mathrm{HCV}$ & 0.044 & 1.476 & 1.010 & 2.156 & & & & \\
\hline NBNC & 0.100 & 0.772 & 0.567 & 1.051 & & & & \\
\hline Tumor Nodules & 0.000 & 5.308 & 2.474 & 11.390 & 0.000 & 5.956 & 2.554 & 13.892 \\
\hline Cirrhosis (Yes vs. No) & 0.223 & 1.418 & 0.809 & 2.485 & & & & \\
\hline VI (Yes vs. No) & 0.009 & 2.131 & 1.21 & 3.754 & 0.904 & 0.959 & 0.483 & 1.901 \\
\hline Tumor size ( $>=5 \mathrm{~cm}$ vs $<5 \mathrm{~cm}$ ) & 0.012 & 2.053 & 1.169 & 3.604 & 0.058 & 1.885 & 0.978 & 3.634 \\
\hline Edmonson Grade & 0.985 & 0.997 & 0.74 & 1.344 & & & & \\
\hline AFP level (Normal $(<20)$ vs. elevated) & 0.001 & 3.192 & 1.631 & 6.248 & 0.004 & 2.823 & 1.387 & 5.745 \\
\hline RB1 mutation & 0.009 & 2.62 & 1.27 & 5.413 & 0.488 & 1.318 & 0.604 & 2.873 \\
\hline TP53 mutation & 0.769 & 1.094 & 0.602 & 1.987 & & & & \\
\hline CTNNB1 mutation & 0.338 & 0.702 & 0.341 & 1.447 & & & & \\
\hline
\end{tabular}


HCV (HR 1.476, CI 1.010-2.156, $P=0.044)$, vascular invasion (HR 2.131, 95\% CI 1.210-3.754, $P=0.009$ ), AFP $20 \mathrm{ng} / \mathrm{ml}$ (HR 3.192, CI 1.631-6.248, $P=0.001$ ), tumor size (HR 2.053, 95\% CI 1.169-3.604, $P=0.012$ ) and $R B 1$ aberrations (HR 2.62, 95\% CI 1.302-5.228, $P=0.009$ ) (Table 3). In the multivariate analysis, the 5-gene score was not validated as a significant prognostic factor; AFP20ng/ml (HR 2.918, CI 1.423-5.984, $P=.003)$, tumor nodules (HR 6.818, CI 2.873-16.184, $P<0.001$ ), HCV (HR 1.718, CI 1.159-2.546, $P=.007$ ) and tumor size (HR 1.909 , CI $0.984-3.705, P=.056$ ) were found as the significant prognostic factors for early RFS of HCC patients after resection (Table 3).

In this study, we aimed to validate the association of the G1-G6 signature and the prognostic value of the 5gene score in Korean HCC patients. These two molecular signatures showed remarkable concordance between $\mathrm{CNV}$ and the mutation profiles of Korean HCC patients and the patients in French studies [13, 14], except for minor discrepancies. For example, G5 and G6 rates are lower in our cohort than in the original cohort, which seems to be related to the lower rate of CTNNB1 mutation in our cohort. According to the 5-gene score, the poor prognosis group showed shorter disease-specific survival and early recurrence-free survival as well as a significant association with microvascular invasion, tumor size, high AFP levels, and TP53 mutations.

However, the 5-gene score was not an independent prognostic factor for the survival of HCC patients. This may be due to the low event rate [only 30 patients out of 205 (14.6\%) died during follow-up, in contrast to French data, in which 106/314 (33.8\%) died during follow-up], which may have resulted in different multivariable outcomes.

\section{Conclusions}

Thus, our analysis suggests that G1-G6 and 5-gene signatures are in concordance between genetic profiles of Korean HCC patients and patients in original French studies. Therefore, in the future, by combining all of these cohorts, we may be able to assertively establish the clinical and pathological relevance of the 5-gene score and develop therapeutic strategies for $\mathrm{HCC}$ patients worldwide.

\section{Abbreviations \\ CNV: Copy Number Variation; DSS: Disease-Specific Survival; HCC: Hepatocellular Carcinoma; LOH: Loss Of Heterozygosity; RFS: Recurrence-Free Survival}

\section{Acknowledgements}

The bio specimen and data used in this study was provided by Asan Bio-Resource Center, Korea, Korea Biobank Network (2012-8 (51)). Institut National de la Santé et de la Recherche Médicale (INSERM) generated transcriptomic and 5-gene score signatures.

\section{Funding}

This research was supported by a grant of the Korea Health Technology R\&D Project through the Korea Health Industry Development Institute, funded by the Ministry of Health \& Welfare (HI6C1985) and by Basic Science Research Program through the National Research Foundation of Korea funded by the Ministry of Science, ICT and future Planning(NRF-2015R1A2A2A04004063).

\section{Availability of data and materials}

The whole exome sequencing data was uploaded to cbioportal (http:// www.cbioportal.org/study?id=lihc_amc_prv) as described in our previous study [5]. CytoscanHD data was uploaded to Gene Expression Omnibus database (accession number: GSE54504).

\section{Authors' contributions}

SMA, JZR, EY contributed to conception and design of the study, and revised the manuscript. FH contributed to analysis, interpretation and writing of the manuscript. IP and JCN contributed in the analysis and revising manuscript. All authors of the study have read and approved the manuscript.

\section{Ethics approval and consent to participate}

The institutional review board of ASAN, South Korea, approved all samples, along with documented consent from all patients who participated in the study (2012-0389).

\section{Consent for publication \\ Not applicable}

\section{Competing interests}

The authors declare that they have no competing interests.

\section{Publisher's Note}

Springer Nature remains neutral with regard to jurisdictional claims in published maps and institutional affiliations.

\section{Author details \\ ${ }^{1}$ Department of Genome Medicine and Science, College of Medicine, Gachon University, Seongnam, South Korea. ${ }^{2}$ Department of \\ Hematology-Oncology, Gachon University Gil Hospital, Incheon, South Korea. ${ }^{3}$ Department of Biosciences, Cancer Genetics and Epigenetics Lab, COMSATS Institute of Information Technology, Islamabad, Pakistan. ${ }^{4}$ Inserm, UMR-1162, Génomique Fonctionnelle des Tumeurs Solides, Équipe Labellisée Ligue Contre le Cancer, 27 rue Juliette Dodu, F-75010 Paris, France. ${ }^{5}$ Labex Immuno-Oncology, Sorbonne Paris Cité, Faculté de Médecine, Université Paris Descartes, Paris, France. ${ }^{6}$ Sorbonne Paris Cité, UFR SMBH, Université Paris 13, F-93000 Bobigny, France. ${ }^{7}$ Université Paris Diderot, F-75013 Paris, France. ${ }^{8}$ Department of Pathology, University of Ulsan College of Medicine, Asan Medical Center, 88, OLYMPIC-RO 43-GIL, SONGPA-GU, SEOUL 138-736, South Korea.}

Received: 29 January 2017 Accepted: 6 March 2018

Published online: 18 May 2018

References

1. McGlynn KA, London WT. The global epidemiology of hepatocellular carcinoma, present and future. Clin. Liver Dis. 2011;15(2): 223-X.

2. Forner A, Gilabert M, Bruix J, Raoul J-L. Treatment of intermediate-stage hepatocellular carcinoma. Nat Rev Clin Oncol. 2014;11(9):525-35.

3. Llovet JM, Burroughs A, Bruix J. Hepatocellular carcinoma. Lancet. 2003; 362(9399):1907-17.

4. Llovet JM, et al. Sorafenib in advanced hepatocellular carcinoma. N Engl J Med. 2008:359(4):378-90

5. Ahn S-M, et al. Genomic portrait of resectable hepatocellular carcinomas: implications of RB1 and FGF19 aberrations for patient stratification. Hepatology. 2014;60(6):1972-82.

6. Dong H, Qian Z, Zhang L, Chen Y, Ren Z, Ji Q. Genomic and transcriptome profiling identified both human and HBV genetic variations and their interactions in Chinese hepatocellular carcinoma. Genomics Data. 2015;6:1-3.

7. Fujimoto A, et al. Whole-genome sequencing of liver cancers identifies etiological influences on mutation patterns and recurrent mutations in chromatin regulators. Nat Genet. 2012:44(7):760-4.

8. Guichard C, et al. Integrated analysis of somatic mutations and focal copy-number changes identifies key genes and pathways in hepatocellular carcinoma. Nat Genet. 2012;44(6):694-8. 
9. Kan Z, et al. Whole-genome sequencing identifies recurrent mutations in hepatocellular carcinoma. Genome Res. 2013;23(9):1422-33.

10. Lee J-S, et al. Classification and prediction of survival in hepatocellular carcinoma by gene expression profiling. Hepatol Baltim Md. 2004;40(3):667-76.

11. Lee J-S, et al. A novel prognostic subtype of human hepatocellular carcinoma derived from hepatic progenitor cells. Nat Med. 2006;12(4):410-6.

12. Hoshida Y, et al. Gene expression in fixed tissues and outcome in hepatocellular carcinoma. N Engl J Med. 2008;359(19):1995-2004.

13. Boyault $\mathrm{S}$, et al. Transcriptome classification of HCC is related to gene alterations and to new therapeutic targets. Hepatology. 2007:45(1):42-52.

14. Nault J, et al. A hepatocellular carcinoma 5-gene score associated with survival of patients after liver resection. Gastroenterology. 2013;145(1):176-87.

15. Woo HG, et al. Association of TP53 mutations with stem cell-like gene expression and survival of patients with hepatocellular carcinoma. Gastroenterology. 2011;140(3):1063-1070.e8.

16. Schulze $K$, et al. Exome sequencing of hepatocellular carcinomas identifies new mutational signatures and potential therapeutic targets. Nat Genet. 2015;47(5):505-11.

17. Cibulskis $\mathrm{K}$, et al. Sensitive detection of somatic point mutations in impure and heterogeneous cancer samples. Nat Biotechnol. 2013;31(3):213-9.

18. Olshen AB, Bengtsson H, Neuvial P, Spellman PT, Olshen RA, Seshan VE. Parent-specific copy number in paired tumor-normal studies using circular binary segmentation. Bioinforma Oxf Engl. 2011;27(15):2038-46.

\section{Submit your next manuscript to BioMed Central and we will help you at every step:}

- We accept pre-submission inquiries

- Our selector tool helps you to find the most relevant journal

- We provide round the clock customer support

- Convenient online submission

- Thorough peer review

- Inclusion in PubMed and all major indexing services

- Maximum visibility for your research

Submit your manuscript at www.biomedcentral.com/submit

C) Biomed Central 